\title{
Isospin breaking corrections to the HVP at the physical point
}

\author{
Vera Gülpers ${ }^{* 1,2}$, Andreas Jüttner ${ }^{1}$, Christoph Lehner ${ }^{3}$, Antonin Portelli ${ }^{2}$ \\ ${ }^{1}$ School of Physics and Astronomy, University of Southampton, Southampton SO17 1BJ, UK \\ ${ }^{2}$ School of Physics and Astronomy, University of Edinburgh, Edinburgh EH9 3JZ, UK \\ ${ }^{3}$ Physics Department, Brookhaven National Laboratory, Upton, NY 11973, USA \\ E-mail: Vera.Guelpers@ed.ac.uk
}

\section{RBC and UKQCD Collaborations}

A determination of the hadronic vacuum polarization contribution to the anomalous magnetic moment of the muon from lattice QCD aiming at a precision of $1 \%$ requires to include isospin breaking corrections in the computation. We present a lattice calculation of the QED and strong isospin breaking corrections to the hadronic vacuum polarization with Domain Wall fermions. The results are obtained using quark masses which are tuned such that pion and kaon masses agree with their physical values including isospin breaking corrections.

The 36th Annual International Symposium on Lattice Field Theory - LATTICE2018 22-28 July, 2018

Michigan State University, East Lansing, Michigan, USA.

\footnotetext{
* Speaker.
} 


\section{Introduction}

The current most precise determination of the hadronic vacuum polarization (HVP) contribution to the anomalous magnetic moment of the muon $a_{\mu}$ is obtained using the cross section of $e^{+} e^{-} \rightarrow$ hadrons (see $[1,2,3]$ for recent results) and has an error of about $\lesssim 1 \%$. A lattice calculation aiming at a similar precision requires to include isospin breaking corrections.

In nature, isospin symmetry is broken by the different quark masses of the up and the down quark and their different electric charges. These effects are expected to be of the order $\mathscr{O}\left(\left(m_{d}-\right.\right.$ $\left.\left.m_{u}\right) / \Lambda_{\mathrm{QCD}}\right)$ and $\mathscr{O}(\alpha)$, respectively. In this proceedings we present a calculation of isospin breaking corrections to the hadronic vacuum polarization at physical quark masses. These results have been published in [4] and are a continuation of our work in [5], where we calculated isospin breaking correction to the HVP at unphysical quark masses. Other calculations of isospin breaking corrections to the HVP can be found in [6, 7].

The structure of the proceedings is as follows: In section 2 we give details on the computational setup and describe our procedure to tune the quark masses to their physical values including isospin breaking corrections. In section 3 we discuss our results for QED and strong isospin breaking corrections. Conclusions and outlook are given in section 4.

\section{Computational Setup and Tuning of the Quark Masses}

In this work we calculate isospin breaking corrections using an expansion $[8,9]$ around the isospin symmetric limit, i.e.

$$
C(t)=C^{0}(t)+\alpha C^{\mathrm{QED}}(t)+\sum_{f} \Delta m_{f} C^{\Delta m_{f}}(t)+O\left(\alpha^{2}, \alpha \Delta m, \Delta m^{2}\right)
$$

for a correlation function $C(t)$, where $C^{0}(t)$ is the correlation function in the isospin symmetric case, $\alpha C^{\mathrm{QED}}(t)$ and $\sum_{f} \Delta m_{f} C^{\Delta m_{f}}(t)$ are the leading order QED and strong isospin breaking correction, respectively.

The set of diagrams at $O(\alpha)$ from the expansion in the electromagnetic coupling is shown in figure 1. These can be divided in three different classes of diagrams: QED corrections to the quarkconnected contribution are given by diagrams $V$ and $S$, QED corrections to the quark-disconnected contribution are given by diagrams $F$ and $D 3$. Diagrams $T, D 1, D 2$ and $T_{d}, D 1_{d}, D 2_{d}$ are electromagnetic effects for the sea quarks for the quark-connected and quark-disconnected contribution, respectively. In this work we calculate the connected diagrams $(V, S)$ and the leading disconnected diagram $F$. All other diagrams are at least $1 / N_{c}$ or $S U(3)$ flavour suppressed for the HVP and we add an overall systematic uncertainty of $30 \%$ of the QED correction from neglecting these diagrams on our final result for $a_{\mu}$ in [4]. Note, that we use local vector currents renormalized by $Z_{V}$ for the photon insertions, and thus, tadpole contributions are absent. We use Feynman gauge and the $\mathrm{QED}_{L}[10]$ prescription for the photon propagators

$$
\Delta_{\mu v}(x-y)=\delta_{\mu v} \frac{1}{N} \sum_{k, \vec{k} \neq 0} \frac{e^{i k \cdot(x-y)}}{\hat{k}^{2}} .
$$




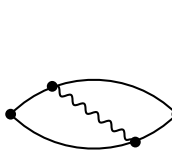

(a) $\mathrm{V}$

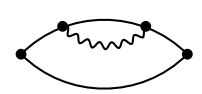

(b) $\mathrm{S}$

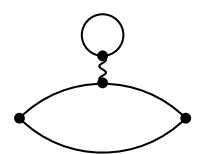

(c) $\mathrm{T}$

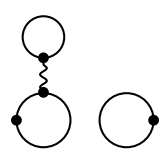

(d) $\mathrm{T}_{d}$

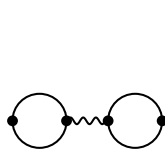

(e) $\mathrm{F}$

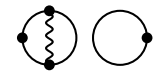

(f) D3

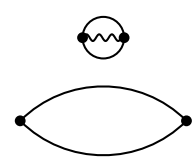

(g) D1

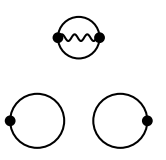

(h) $\mathrm{D} 1_{d}$

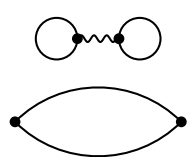

(i) D2

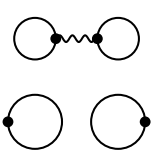

(j) $\mathrm{D} 2_{d}$

Figure 1: QED correction diagrams at $O(\alpha)$.

Isospin breaking corrections due to the expansion in the quark masses in equation (2.1) are given by the diagrams in figure 2. In this work, we only calculate the quark-connected correction (diagram $M$ ). We neglect mass corrections to the quark-disconnected contribution (diagram $O$ ), which are $1 / N_{c}$ and $S U(3)$ flavour suppressed and assign an additional 10\% of the strong isospin breaking correction as an systematic error in the final result for $a_{\mu}$ [4]. The mass correction to the sea quarks (diagram $R$ and $R_{d}$ for the quark-connected and quark-disconnected HVP, respectively) is proportional to a factor of $\left(\Delta m_{u}+\Delta m_{d}\right)$ and we find $\Delta m_{u} \approx-\Delta m_{d}$ when tuning the quark masses to their physical values and thus expect diagram $R$ to be negligible.

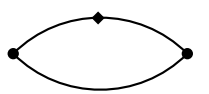

(a) $\mathrm{M}$

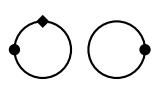

(b) $\mathrm{O}$

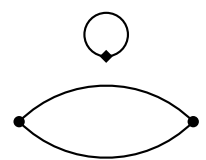

(c) $\mathrm{R}$

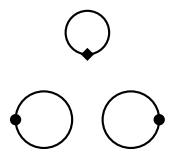

(d) $R_{d}$

Figure 2: Strong Isospin Breaking correction diagrams at $O(\Delta m)$.

We calculate the isospin breaking corrections to the HVP on a $48^{3} \times 96$ lattice using $N_{f}=$ $2+1$ dynamical flavours of Mobius Domain Wall Fermions on a single lattice spacing $a^{-1}=$ $1.730(4) \mathrm{GeV}$. The isospin symmetric calculation is done using a light and a strange quark mass, that have been tuned to reproduce a pion of $m_{\pi}^{0}=135.0 \mathrm{MeV}$ and a kaon of $m_{K}^{0}=495.7 \mathrm{MeV}$ in the absence of QED and strong isospin breaking effects [11]. To obtain up, down and strange quark masses at their physical values including QED, we proceed as follows. We fix the charged pion, neutral kaon and charged kaon masses including QED to their experimental values

$$
\begin{aligned}
& a m_{\pi^{+}}^{\exp }=\left[m_{\pi}^{0}+\alpha m_{\pi^{+}}^{\mathrm{QED}}+\Delta m_{d} m_{\pi^{+}}^{\Delta m_{d}}+\Delta m_{u} m_{\pi^{+}}^{\Delta m_{u}}\right] \\
& a m_{K^{+}}^{\exp }=\left[m_{K}^{0}+\alpha m_{K^{+}}^{\mathrm{QED}}+\Delta m_{u} m_{K^{+}}^{\Delta m_{u}}+\Delta m_{s} m_{K^{+}}^{\Delta m_{s}}\right] \\
& a m_{K^{0}}^{\exp }=\left[m_{K}^{0}+\alpha m_{K^{0}}^{\mathrm{QED}}+\Delta m_{d} m_{K^{0}}^{\Delta m_{d}}+\Delta m_{s} m_{K^{0}}^{\Delta m_{s}}\right]
\end{aligned}
$$

where $m_{H}^{0}$ is the isospin symmetric mass of $H, \alpha m_{H}^{\mathrm{QED}}$ the QED correction to mass of $H$ and $\Delta m_{f} m_{H}^{\Delta m_{f}}$ the correction to the mass of $H$ from a shift of the quark mass $\Delta m_{f}=\left(m_{f}-m_{f}^{0}\right)$. The 
shifts $\Delta m_{f}$ in the quark masses are free parameters that can be tuned after all the required correlation functions have been calculated. Once having tuned the quark masses to reproduce the physical values of $\pi^{+}, K^{+}$and $K^{0}$ we checked that we also correctly reproduce the neutral pion mass.

In addition, the tuning of the quark masses as in equations (2.3) - (2.5) requires to determine the lattice spacing in the presence of QED. Here, we choose to set the lattice spacing by fixing the mass of the $\Omega^{-}$baryon

$$
a \rightarrow a\left(\Delta m_{s}\right)=\left(m_{\Omega}^{0}+\alpha m_{\Omega}^{\mathrm{QED}}+3 \Delta m_{s} m_{\Omega}^{\Delta m_{s}}\right) / m_{\Omega}^{\exp } .
$$

We find the shift in the lattice spacing to be smaller then the statistical error on the lattice spacing and therefore neglect this effect in the following.

\section{Results}

In the following we discuss our results for different contributions of the QED and strong isospin breaking corrections to the hadronic vacuum polarization. The HVP contribution to the anomalous magnetic moment of the muon can be calculated from the vector-vector two-point function $[12,13]$

$$
a_{\mu}=\sum_{t} w_{t} C(t) \quad \text { with } \quad C(t)=\frac{1}{3} \sum_{j=0}^{2} \sum_{\vec{x}}\left\langle J_{j}(\vec{x}, t) J_{j}(0)\right\rangle
$$

where $J_{j}=\frac{2}{3} \bar{u} \gamma_{j} u-\frac{1}{3} \bar{d} \gamma_{j} d+\cdots$ are electromagnetic vector currents. In this work we use local vector currents multiplied with the vector current renormalization $Z_{V}$. We also calculate the QED correction to $Z_{V}$ and find this to be negligible for our setup.

\subsection{Quark-connected QED correction}

Our data for the quark-connected QED correction (diagrams $V$ and $S$ in figure 1) to the integrand $w_{t} C(t)$ is shown in figure 3. The QED correction to $a_{\mu}$ from these contributions can then be obtained by integrating the data over the euclidean time $t$. However, as one can see in figure 3 the statistical error on the data is large. Therefore, we replaced the data in the integration for $a_{\mu}$ by a fit ansatz

$$
C(t)=\left(c_{1}+c_{0} t\right) e^{-E t} .
$$

We fix $E$ to the energy of the lowest lying state, which, including QED, is given by $\pi \gamma$, where in $\mathrm{QED}_{L}[10]$ the photon has one unit of momentum (cf. equation (2.2)). We then fit our data to the ansatz (3.2) using $c_{1}$ and $c_{2}$ as free parameters. The result of this fit is shown in figure 3 by the solid line.

For the connected QED correction to $a_{\mu}$ we find

$$
a_{\mu}^{\mathrm{QED}, \mathrm{con}}=5.9(5.7)_{S}(1.1)_{E}(0.3)_{C}(1.2)_{V}(0.0)_{A}(0.0)_{Z} \times 10^{-10},
$$

where the first error ()$_{S}$ is statistical. Further to that, we have assigned the following systematic errors. A systematic error from our fit ansatz ()$_{E}$ is determined by varying the input for the lowest energy between $\pi \gamma$ and $\pi \pi$. We estimate a discretization error ()$_{C}$ as $(a \Lambda)^{2}$ with $\Lambda=400 \mathrm{MeV}$. 


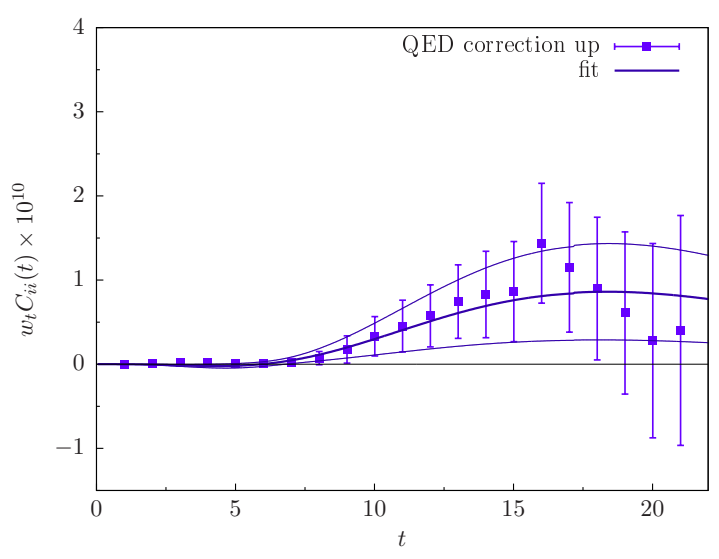

Figure 3: Results for the quark-connected QED correction to $w_{t} C(t)$. The result of the fit is shown by the solid line, with the fainter lines indicating the error band of the fit.

When including QED in a lattice calculation, finite volume corrections can be large (see e.g. [14]). In this work, we estimate finite volume corrections, be replacing the photon propagator by its infinite volume expression and take the difference in the final result as a systematic error ()$_{V}$ from not correcting for these effects. More details on using an infinite volume photon propagator can be found in the supplementary material of [4]. However, a study presented at this conference [15] suggests, that the finite volume effects for the QED correction to the HVP are much smaller. Finally, we propagate uncertainties from the lattice spacing ()$_{A}$ and vector renormalization constant ()$_{Z}$, but find them to be negligible compared to other sources of systematic errors.

\subsection{Quark-disconnected QED correction}

The leading QED correction to the quark-disconnected HVP is given by diagram $F$ in figure 1 . Here, we are only interested in contributions, where in addition to the photon the quark lines are connected by gluons. If no additional gluons connect both quark lines, these contributions are conventionally counted as higher order HVP contributions and need to be subtracted in this context.

We calculated the leading quark-disconnected QED correction to the HVP using data generated for the light-by-light scattering project [16]. The data is fitted using the same ansatz (3.2) as for the connected QED correction. We find for the leading quark-disconnected QED correction to the anomalous magnetic moment

$$
a_{\mu}^{\mathrm{QED}, \text { disc }}=-6.9(2.1)_{S}(1.3)_{E}(0.4)_{C}(0.4)_{V}(0.0)_{A}(0.0)_{Z} \times 10^{-10},
$$

where we have assigned a similar set of systematic errors as above.

\subsection{Strong Isospin breaking corrections}

In figure 4 we show the correction to the vector two-point function $C(t)$ for the quark-connected mass insertion diagram (diagram $M$ in figure 2). We fit the correlator using a similar ansatz (3.2) as for QED, using $\pi \pi$ as the lowest lying energy state as an input. Here, we vary the energy between two interacting and two free pions to obtain a systematic error ()$_{E}$ from the fit ansatz. 


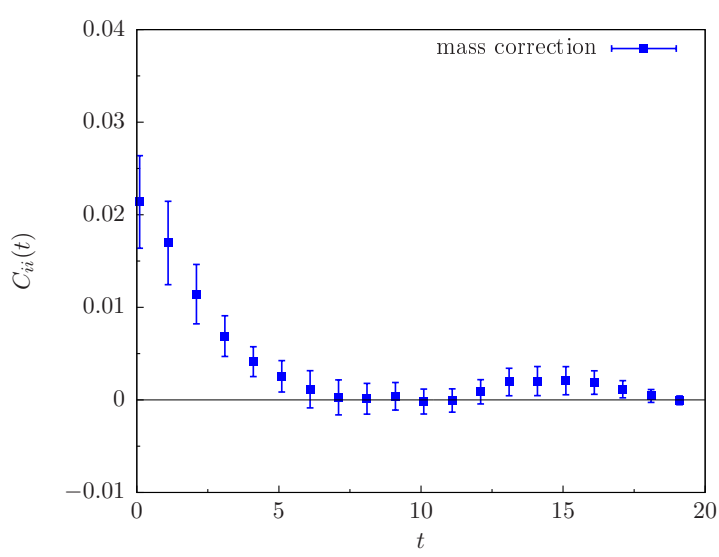

Figure 4: The isospin breaking correction to $C(t)$ from the connected mass insertion diagram $M$.

For the correction to $a_{\mu}$ from diagram $M$ we find

$$
a_{\mu}^{\mathrm{sIB}}=10.6(4.3)_{S}(1.3)_{E}(0.6)_{C}(6.6)_{V}(0.1)_{A}(0.0)_{Z} \times 10^{-10} .
$$

For the strong isospin breaking correction we estimate finite volume corrections using chiral perturbation theory [17].

\section{Conclusions}

The results shown here are part of RBC/UKQCD's recently published result for the anomalous magnetic moment of the muon [4]. This work is the first calculation of the HVP contribution to the $a_{\mu}$ including a calculation of QED and strong isospin breaking corrections directly at the physical point. Although our statistical errors are still large, we find isospin breaking corrections to be at the order of $1 \%$, with results for the connected QED correction given in (3.3), the leading disconnected QED correction given in (3.4) and the connected strong isospin breaking correction given in (3.5). For the future, we plan to increase the statistics on these contributions in addition to calculating the missing quark-disconnected diagrams and studying the effect of isospin breaking corrections for the sea quarks. In that context we are looking into reusing the data from the hadronic light-by-light scattering project [16] for all of the diagrams shown in figure 1. Further to that, we will extend our study of isospin breaking corrections to a second lattice spacing in oder to take a continuum limit.

These future improvements will allow us to determine the isospin breaking correction to $a_{\mu}^{H V P}$ precise enough to be consitent with reaching an overall error of $<1 \%$ on the total QCD + QED result for $a_{\mu}^{H V P}$ from a lattice calculation.

\section{Acknowledgments}

V.G. and A.P. received funding from the European Research Council (ERC) under the European Union's Horizon 2020 research and innovation programme under grant agreement No 757646. A.P also received funding from UK STFC grants ST/L000458/1 and ST/P000630/1. C.L. is supported by US DOE Contract DESC0012704(BNL) and by a DOE Office of Science Early CareerAward. V.G. and A.J. received funding from STFC consolidated grant ST/P000711/1. A.J. received 
funding from the European 618 Research Council under the European Union's Seventh Framework Program (FP7/2007-619 2013) / ERC Grant agreement 279757. This work was supported by resources provided by the Scientific Data and Computing Center (SDCC) at Brookhaven National Laboratory (BNL), a DOE Office of Science User Facility supported by the Office of Science of the US Department of Energy. The SDCC is a major component of the Computational Science Initiative at BNL. We gratefully acknowledge computing resources provided through USQCD clusters at Fermilab and Jefferson Lab. This work was also supported by the DiRAC Blue Gene Q Shared Petaflop system at the University of Edinburgh, operated by the Edinburgh Parallel Computing Centre on behalf of the STFC DiRAC HPC Facility (www.dirac.ac.uk). This equipment was funded by BIS National E-infrastructure capital grant ST/K000411/1, STFC capital grant ST/H008845/1, and STFC DiRAC Operations grants ST/K005804/1 and ST/K005790/1. DiRAC is part of the UK National E-Infrastructure.

\section{References}

[1] A. Keshavarzi et al., Phys. Rev. D97, 114025 (2018), [arXiv:1802.02995]

[2] F. Jegerlehner, (2017), arXiv:1705.00263

[3] M. Davier et al., Eur. Phys. J. C77, 827 (2017), [arXiv:1706.09436]

[4] T. Blum et al., Phys. Rev. Lett. 121, 022003 (2018), [arXiv:1801.07224]

[5] P. Boyle et al., JHEP 09, 153 (2017), [arXiv:1706.05293].

[6] D. Giusti et al., JHEP 10, 157 (2017), arXiv:1707.03019 [hep- lat]

[7] B. Chakraborty et al., Phys. Rev. Lett. 120, 152001 (2018), [arXiv:1710.11212]

[8] G. M. de Divitiis et al., JHEP 04, 124 (2012), [arXiv:1110.6294]

[9] G. M. de Divitiis et al., Phys. Rev. D87, 114505 (2013), [arXiv:1303.4896]

[10] M. Hayakawa and S. Uno, Prog. Theor. Phys. 120, 413 (2008), [arXiv:0804.2044]

[11] T. Blum et al., Phys. Rev. D93, 074505 (2016), [arXiv:1411.7017]

[12] D. Bernecker and H. B. Meyer, Eur. Phys. J. A47, 148 (2011), [arXiv:1107.4388]

[13] X. Feng et al., Phys.Rev. D88, 034505 (2013), [arXiv:1305.5878]

[14] S. Borsanyi et al., Science 347, 1452 (2015), [arXiv:1406.4088]

[15] J. Harrison et al., these proceedings

[16] T. Blum et al., Phys. Rev. Lett. 118, 022005 (2017), [arXiv:1610.04603]

[17] J. Bijnens and J. Relefors, JHEP 12, 114 (2017), arXiv:1710.04479 [hep-lat] 